\title{
Superconductivity in the 2D Hubbard model: yes, no, or maybe?
}

\author{
Absence of superconductivity in the pure two-dimensional Hubbard Model \\ Authors: Mingpu Qin, Chia-Min Chung, Hao Shi, Ettore Vitali, Claudius Hubig, \\ Ulrich Schollwock, Steven R. White, and Shiwei Zhang (Simons Collaboration on the \\ Many-Electron Problem) \\ Phys. Rev. X 10, 031016 (2020)

\section{Recommended with a Commentary by Andrey V Chubukov, University of Minnesota}

This paper generated some reaction solely based on its title, and the purpose of this commentary is to discuss its actual content. As one could anticipate, the goal of Mingpu Qin et al was to give a definite answer to a well-posed question whether the ground state of the pure 2D Hubbard model with nearest-neighbor hopping $t$ on a square lattice, and on-site interaction $U$, is a superconductor. For this, Mingpu Qin et al studied a linear response to the $d_{x^{2}-y^{2}}$ pairing field and the pair-pair correlation function by combining two state-of-the-art computational methods - constrained path auxiliary field Quantum Monte Carlo and density matrix renormalization group. They obtained very similar results using both methods, which allowed them to draw the conclusions with high degree of confidence. Simulations have been done on rectangular lattices of size $N=L_{x} L_{y}$ in cylinder geometry with periodic boundary conditions along the $\mathrm{y}$ direction and open boundary conditions along the $\mathrm{x}$ direction They varied the aspect ratios of the cylinders to make sure that the rectangular cells do not impact their results.

The key message of this paper is that for $U / t$ around $6-8$ and doping $0.1<x<0.2$, the ground state is not a superconductor but rather has a stripe order. They demonstrated quite convincingly that a superconducting order parameter decays exponentially at deviations from the bonds, to which they applied a pairing field. The pair-pair correlation function also decays exponentially. They analyzed the evolution of the amplitudes of stripe and superconducting (pairing) orders upon varying the applied pairing field $h_{p}$, and found that at $h_{p} \rightarrow 0$, stripe order saturates at a finite value, while superconducting order vanishes. A stripe order in the same range of parameters has been earlier reported in [1]. An earlier study of the $t$-only Hubbard model in the same range of $U$ and $x$ (Ref.[7]) did find $d_{x^{2}-y^{2}}$ superconductivity but didn't analyze a possibility that it gets destroyed by a stripe order.

The authors wrote explicitly at the end of Introduction: "We emphasize that our work does not imply a general statement that there is no superconducting order anywhere in the pure Hubbard model. Rather, our focus is on the nature of the pairing order in the pure Hubbard model in the physically important parameter regime as a model for cuprate superconductors." 
In view of this last statement, I think it may be useful for a reader if I try to place this work into a broader context of superconductivity in a Hubbard model with $t-t^{\prime}$ hoping, at various dopings and various $U / t$. This issue has been studied by many (!) authors (see, e.g. Ref. [2] and references therein), and much progress has been achieved both analytically and numerically, using recent advances of the computational tools

First, it is well understood that Hubbard repulsion is not an obstacle to superconductivity with gap symmetry different from an ordinary $s$-wave. In short, the pairing interaction get dressed by fermionic particle-hole pairs, becomes coordinate-dependent, and undergoes Friedel-type oscillations at large distances, i.e., it occasionally becomes attractive.

Second, there exist certain limits, where superconductivity can be analyzed rigorously by expanding in a small parameter, although relevant energy scales can be very small. One such limit is a small density of electrons, $1-x \ll 1$ (not to be confused with small

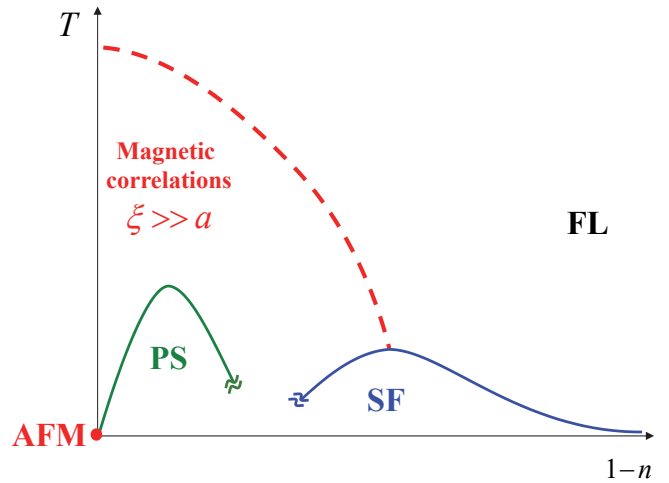

Figure 1: Schematic phase diagram of the repulsive $t$-only $2 \mathrm{D}$ Hubbard model near half-filling. The abbreviations are Fermi liquid (FL), superfluid (SF), antiferromagnet (AFM), and phase separation (PS). Borrowed from Ref. [6]. In the notations used here, SF is a superconductor, and PS corresponds to stripe order. doping $x$ away from half-filling). In this limit, one can use density as an expansion parameter while keeping $U / t$ arbitrary. The result is that the Hubbard model is a superconductor [5], but the symmetry of the pairing state is not $d_{x^{2}-y^{2}}$. Rather, it is $d_{x y}$ at small $t^{\prime} / t$ and more exotic $g$-wave at larger $t^{\prime} / t$. Superconductivity in this limit is not caused by any pairing boson and originates from a pure Kohn-Luttinger effect. Another is a weak coupling limit $U / t \ll 1$. Here, the ground state is again a superconductor, but the pairing symmetry depends on the electron density. Reasonably close to half-filling, it is $d_{x^{2}-y^{2}}$ for all $t^{\prime}>t$ simply because the angle-dependent fermionic density of states peaks near the corners of the Brillouin zone, where $d_{x^{2}-y^{2}}$ order parameter is the largest. And the third limit is half-filling at $t^{\prime}=0$. Here, the ground state is an antiferromagnet, rather than a superconductor. This holds for all $U / t$, except for very large ones, where the magnetic order becomes ferromagnetic.

Third, in a range of dopings/interactions, where the system behaves as a metal, $d_{x^{2}-y^{2}}$ pairing is often rationalized as coming from the exchange of spin fluctuations, peaked at or near $(\pi, \pi)$ (this is particularly true for hole-like Fermi surface at a finite $t^{\prime}$ ). However, spin fluctuations are good for $d_{x^{2}-y^{2}}$ pairing only in a paramagnetic phase. Once the system develops an antiferromagnetic order, or some other order, which can be thought of as a modification of antiferromagnetism, this order competes with superconductivity and can eliminate it.

Fourth, at large enough $U / t$, electronic excitations become localized, and the system becomes a Mott insulator. Magnetic order is compatible with the insulating state, but superconductivity is not. By this argument, one should expect that superconductivity must become weaker with increasing $U$ and disappear above some doping-dependent $U / t$.

My take is that the results by Mingpu Qin et al fit into this general framework. One way to understand a stripe order, which goes back to Shraiman-Siggia analysis [3], is to view 
it as a result of an instability of a spiral magnetic order at a finite doping. This is also in line with Emery-Kivelson-Lin idea of phase separation [4]. By that token, a stripe order is a competitor to superconductivity in the same was as an antiferromagnetic order. Given that the ground state of the $t$-only model at half-filling is an antiferromagnet, it seems natural to expect that a competing order does prevent superconductivity, even when this order evolves from antiferromagnetism to stripe order. We illustrate it by Fig. 1, borrowed from Ref.[6] At small $U / t$ a competing order exists only very near half-filling, where particlehole susceptibility is singular, and $d_{x^{2}-y^{2}}$ superconductivity does emerge at larger $x>x_{c r, 1}$. However, in the $t$-only model, such pairing only holds at $x$ smaller than some $x_{c r, 2}$. As $U / t$ increases, the range $x_{c r, 1}<x<x_{c r, 2}$ shrinks, and eventually must disappear. Mingpu Qin et al probed specifically $d_{x^{2}-y^{2}}$ order, and came to a definite conclusion that it looses to a competitive stripe order for $U / t$ around $6-8$. At smaller $U / t \leq 4$, they hinted that $d_{x^{2}-y^{2}}$ superconductivity may develop, as diagrammatic Monte-Carlo studies suggest [5].

Mingpu Qin et al also discussed an interesting possibility of a co-existence of superconductivity and stripe order. They argued that for their parameters this does not happen. Very recent variational Monte-Carlo study for $U / t=8$ (Ref. [8]) confirmed the absence of a co-existence and found a stripe order up to $x \leq 0.2$. Mingpu Qin et al hinted that stripe and

$d_{x^{2}-y 2}$ orders may co-exist for smaller $U$. This is an interesting question, which will certainly be addressed in future studies.

\section{References}

[1] B.-X. Zheng et al, Science 358, 1155 (2017). See also A. Wietek et al, arXiv:2009.10736.

[2] D. J. Scalapino, Rev. Mod. Phys. 84, 1383 (2012).

[3] B. Shraiman and E. Siggia, Phys. Rev. Lett. 62, 1565 (1989); Th. Dombre. J. Phys. France 51, 847-856 (1990).

[4] V. J. Emery, S. A. Kivelson, and H. Q. Lin, Phys. Rev. Lett. 64, 475 (1990).

[5] R. Hlubina, PRB 59, 9600 (1999); Y. Deng, E. Kozik, N. V. Prokof'ev, and B. V. Svistunov, Europhys. Lett. 110, 57001 (2015).

[6] Fedor Šimkovic IV., Youjin Deng, N. V. Prokof'ev, B. V. Svistunov, I. Tupitsyn, and E. Kozik Phys. Rev. B 96, 081117 (2017).

[7] E. Gull and A. J. Millis, Phys. Rev. B 90, 041110(R) (2014).

[8] S. Sorella, arXiv 2101.07045. 